NASA Technical Memorandum 89898

\title{
Automated Measurement of the Bit-Error Rate as a Function of Signal-to-Noise Ratio for Microwave Communications Systems
}

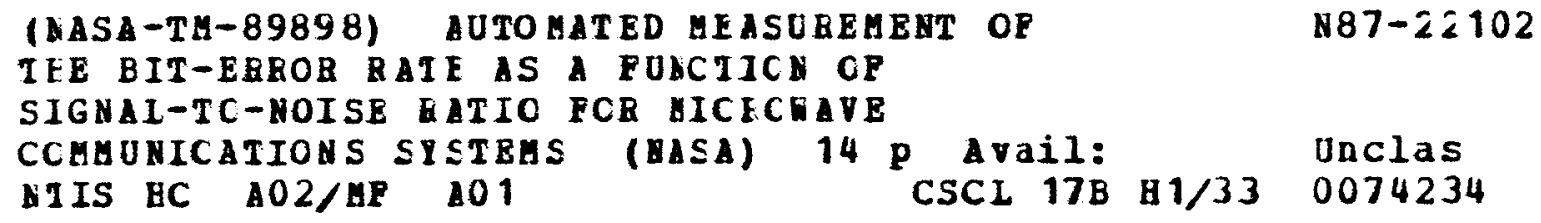

Robert J. Kerczewski, Elaine S. Daugherty, and Ihor Kramarchuk Lewis Research Center

Cleveland, Ohio

Prepared for the 29th Automatic RF Techniques Group Conference sponsored by the Institute of Electrical and Electronics Engineers Las Vegas, Nevada, June 12-13, 1987 


\title{
AUTOMATED MEASUREMENT OF THE BIT-ERROR RATE AS A FUNCTION OF SIGNAL-
}

\section{TO-NOISE RATIO FOR MICROWAVE COMMUNICATIONS SYSTEMS}

\author{
Robert J. Kerczewski, Elaine S. Daugherty, and Ihor Kramarchuk \\ National Aeronautics and Space Administration \\ Lewis Research Center \\ Cleveland, Ohio 44135
}

\begin{abstract}
The performance of microwave systems and components for digital data transmission can be characterized by a plot of the bit-error rate as a function of the signal-to-noise ratio (or $E_{b} / N_{0}$ ). Methods for the efficient automated measurement of bit-error rates and signal-to-noise ratios, developed at NASA Lewis Research Center, are described. Noise measurement considerations and time requirements for measurement accuracy, as well as computer control and data processing methods, are discussed.
\end{abstract}

\section{Introduction}

The performance of microwave digital data transmission systems and components can be quantized in terms of the rate at which bit-errors occur. Amplitude and phase distortions in the system, as well as various types of interference, can induce errors. However, errors are primarily a function of the signal-to-noise ratio present at the data demodulator input. A curve of the bit-error rate (BER) as a function of the recelved energy-per-bit to noise ratio $\left(E_{b} / N_{0}\right)$, given the other distortions present, sufficientiy characterizes the performance of the system, allowing operating parameters to be chosen to yield a desired BER.

Work in advanced digital comunications systems at NASA Lewis has created a requirement for the laboratory evaluation of communications systems and system components. To meet this requirement, an automated system for the measurement of BER versus $E_{b} / N_{0}$ has been developed. The system is designed to allow a complete BER curve to be accurately measured in a minimum amount of time.

The major considerations which must be addressed to implement such a system include notse insertion and $E_{b} / N_{0}$ measurement, time requirements for BER measurement accuracy, and the required computer control and data processing algorithms. Approaches which have been successfully applied to these areas are presented and discussed below. 


\section{BER Versus $E_{b} / N_{0}$ Measurement Technique}

The BER is usually measured over a range of values from about $10^{-2}$ to $10^{-7}$ or 10-8. The lower figure is determined by the system performance goal and is chosen so that at least one data point beyond that goal has been taken. For example, if the system is required to perform at a BER of $5 \times 10^{-7}$, the final data point taken must be less than $5 \times 10^{-7}$. This allows the $E_{b} / N_{0}$ required to maintain a BER of $5 \times 10^{-7}$ to be accurately determined by a linear interpolation between the last and next-to-last data points.

A typical measured BER versus $E_{b} / N_{0}$ curve is shown in $F i g .1$. The theoretical (or ideal) curve is usually shown for comparison. Such a curve can be obtained by measuring the BER while varying the $E_{b} / N_{0}$ in 1 dB increments. The starting $E_{b} / N_{0}$ value is determined from the theoretical curve where the $B E R$ is about $10^{-2}$. The $E_{b} / N_{0}$ is incremented by $1 \mathrm{~dB}$ until the measured $B E R$ has exceeded the performance goal, at which time the last data point is taken and the measurement is stopped.

The system for performing this measurement is described by the block diagram shown in $\mathrm{Fig}$. 2. A range of values of $\mathrm{E}_{\mathrm{b}} / \mathrm{N}_{0}$ can be obtained under computer control using a noise insertion system described in the next section. The data generator provides a pseudo-random bit stream to the modulator at the required rate. After the modulated signal has been transmitted through the system under test, the signal (plus noise) is demodulated and the resulting data sequence sent to the data checker. The data checker, after receiving an initial control word, regenerates the original pseudo-random data sequence and compares it, bit by bit, to the data sequence received from the demodulator (Ref. 1). The bit-errors, as well as the total number of bits received, are counted by the data checker. The control computer obtains these counts from the data checker and calculates the bit-error rate. The control computer also monitors signal and noise levels and calculates a table of BER and $E_{b} / N_{0}$ values characterizing the system under test.

\section{Noise Insertion and $E_{b} / N_{0}$ Measurement}

Once a communications system has been placed in service, a single BER measurement characterizes the performance of the system. The signal-to-noise ratio (or energy-per-bit to noise ratio, $E_{b} / N_{0}$ ) is a function of the noise present in the system and the transmitted signal power level and is a fixed quantity. The noise is usually thermal noise in the system plus extraneous noise, such as background noise and interference received by an antenna.

In a laboratory test, the antenna background noise can be eliminated and the thermal nolse can usually be controlled, so that the received $E_{b} / N_{0}$ can be varied by adding white (Gaussian) noise. A noise insertion system such as shown in Fig. 3 (Ref. 2) is used to provide the variable $E_{b} / N_{0}$. The control computer varies the $E_{b} / N_{0}$ in $1 \mathrm{~dB}$ increments through a programmable step attenuator (labeled $A 1$ in $\mathrm{Fig} .3$ ). The second programmable attenuator (A2) controls the output power level. The power monitor port is coupled to the output signal line to allow signal and noise power measurements to be made. Since the notse source produces broadband white noise, a bandpass filter approximating the bandwidth of the system under test is placed ahead of the power meter to improve nolse measurement accuracy. 
The noise insertion can be accomplished at the input to the system under test as well as at the output. For example, in testing a satellite receiver, noise inserted at the input would simulate noise on the satellite uplink. For most measurements, however, the noise is added at the output of the system under test. In this case, measurement of the $E_{b} / N_{0}$ is relatively simple.

A measurement of the signal power is made at the power monitor port by turning off the notse power (setting attenuator $A 1$ set to its maximum setting). Through the control computer, the modulated signal is temporarily turned off and attenuator $A 1$ is returned to its setting for the next measurement point. After the noise power has been measured, the modulated signal is turned on and the BER measured. This process is repeated for each measurement point until the complete $B E R$ versus $E_{b} / N_{0}$ curve has been obtained.

Once the ratio of measured signal power to measured noise power has been calculated, two adjustments are required to yield the $E_{b} / N_{0}$. The first adjustment converts the signal-to-noise ratio to the energy-per-bit to noise ratio. This is done by calculating ratio of the channel bandwidth to the bit rate. This quantity, which represents the difference between the signal power and the energy-per-bit, is then added to the measured signal-to-noise ratio. For example, if a modulated signal at 100 Mbps were being transmitted with a channe 1 bandwidth of $200 \mathrm{MHz}$, the bandwidth to bit rate ratio of 2 (or $3 \mathrm{~dB}$ ) must be added to the measured signal-to-noise ratio to yield $E_{b} / N_{0}$.

The second adjustment to the $E_{b} / N_{0}$ takes into account the bandwidth of the filter on the power monitor port. The ratio of the noise bandwidth of this filter (usually taken as the $3 \mathrm{~dB}$ bandwidth when the filter is 5 th order or higher, Ref. 3) to the channel bandwidth must be added to the $E_{b} / N_{0}$ to adjust for the out-of-channel noise measured during the noise measurement. For example, if the filter on the power monitor port has a bandwidth of $235 \mathrm{MHz}$ while the measurement channel bandwidth is $200 \mathrm{MHz}, 1.175$ (or $0.7 \mathrm{~dB}$ ) must be added to the $E_{b} / N_{0}$. Note that this adjustment is usually not needed for the signal measurement, since the power level of the signal near the band edges is low compared to the band center, while the noise power density is unfform across the band.

When the noise is inserted at the input to the system under test rather than at the output, it is necessary to measure the $E_{b} / N_{0}$ at the input also, particularly if there are nonlinear active devices in the system. The nonlinear devices tend to suppress the noise when the signal is present, yielding a lower actual noise level than is measured with the signal off. The result is a difference between the measured $E_{b} / N_{0}$ and the actual $E_{b} / N_{0}$ at the system output. This error is dependent on the $E_{b} / N_{0}$ at the system input and cannot be measured accurately.

Difficulties in $E_{b} / N_{0}$ measurement occur when interference or spurious signals are present. These signals, which are independent of the modulated signal, will contribute unequally to the noise power measurement and the signal power measurement and thus yield an erroneous $\mathrm{E}_{\mathrm{b}} / \mathrm{N}_{0}$. If the interference was created intentionally (for example, in a test intended to measure the effects of interference on the system), the interfering signals can be removed while a 
baseline measurement is made. The $E_{b} / N_{0}$ values from this baseline measurement are then applied to the subsequent BER measurements made with the interference present, and the $E_{b} / N_{0}$ 's obtained during these subsequent measurements are disregarded. If the interference or spurious signals are inherent in the system and cannot be controlled, a baseline set of $E_{b} / N_{0}$ values can be obtained in a different way. The system under test is removed and replaced with sufficient attenuation or amplification to create a test signal equal in power level to the signal at the output of the system under test. The BER versus $E_{b} / N_{0}$ measurement made with this test signal will provide an adequate set of baseline $E_{b} / N_{0}$ values to apply to BER measurements made on the system with the interference or spurious signals present.

\section{Time Requirements for Accurate Bit-Error Rate Measurement}

The bit-error rate for a digital communications system is primarily a function of the signal-to-noise ratio at the data detector. Since the noise is random in nature, the number of bits checked must be large enough to insure, with a reasonable probability, that the BER measurement is accurate to within a destred error bound.

Applying probability theory to the counting of bit-errors allows calculation of the minimum number of bits required to be checked to obtain a desired BER measurement accuracy. This is done by recognizing the binomial nature of the counting of bit-errors, where each of $n$ received bits has some probability of being in error. Since the probability of a bit being in error is relatively small (less than 0.01), and the number of bits checked is quite large $(>>1000)$, a Poisson approximation to the binomial distribution can be accurately used (Ref. 4). The Potsson approximation yields an expression for the standard deviation which can be used in Chebychev's Inequality to yield the following equation.

where

$$
\operatorname{Probab} 11 \text { ity }\left[n<\frac{1}{(1-P) b E^{2}}\right] \geq P
$$

$n$ is the number of bits checked

$b$ is the actual BER

$P$ is the probability of the measured BER falling within $\pm E$ of the actual BER

for $n$ bits checked

$E$ is the measurement error bound, defined as

$$
E=\frac{\left|b^{\prime}-b\right|}{b} \quad\left(b^{\prime} \text { is the measured } B E R\right)
$$

From the above equation, one can calculate the number of bits, $n$, required to yield a BER measurement that is within a given error bound, $E$, with a probability $P$. The results of several such calculations are plotted in Fig. 4. The number of bits required, $n$, is plotted against the actual BER for three error bounds and three probabilities. For example, when the BER is $10^{-6}$. checking $10^{12}$ bits will result in a measurement error of less than 0.01 for 99 percent of the measurements made. The amount of time required for the 
measurement is obtained by dividing the number of required bits by the data rate.

In order to minimize the time required to measure a complete BER versus $E_{b} / N_{0}$ curve, an algorithm is employed which varies the measurement time based on an initial BER measurement. An example of the algorithm is shown in Fig. 5 . At a data rate of $220 \mathrm{Mbps}$, the algorithm yields an average measurement time of 35 min for a complete curve (BER measured from 10-2 to 10-8) with an error bound of 0.1 and probability of 0.9 .

\section{Computer Control and Data Processing Considerations}

The control computer shown in Fig. 2 is a 32-bit minicomputer. The computer is configured as an Experiment Control and Monitor (EC\&M) Computer, capable of performing numerous RF measurements as well as data transmission tests (Ref. 5). The EC\&M software consists of several libraries of instrument control modules, managed by three phases of control programs, as shown in Fig. 6 .

In the first EC\&M software phase, a menu-driven setup program is used to create two files required to control the test. The test definition file (TDF in Fig. 6) contains all of the necessary information on the instruments and devices to be controlled and monitored during the test, such as device type and address, and limits on device settings or instrument readings. The sequence control file (SDA in Fig. 6) contains the control sequence which accesses the instrument control modules in the proper order.

The second phase consists of a control program and a display program. The control program uses the TDF and SDA files to execute the sequence of actions required to perform the test. The control program also collects and checks the validity of data by comparing it to the limits established in the TDF file, and stores the data in the DAT file (Fig. 6). The display program allows the test engineer to interface to the test in progress, and provides a constantly updating display giving the status of the test being run and the latest set of data collected.

The third phase of the EC\&M control software is the data processing phase. The data file can be printed directly or processed through a separate data processing program to yield the test data in a desired format.

The interaction of the control programs with the various instrument control module libraries for the BER versus $E_{b} / N_{0}$ test is shown in Fig. 7 . The devices to be controlled by the EC\&M for this test (as shown in Figs. 3 and 4) are the data generator, the data checker, two step attenuators, and a power meter.

The data generator and checker are custom built devices controlled by a 6809 microcomputer. The EC\&M communicates with the microcomputer through an RS-232 interface. The data generator need only be commanded to turn on or off at the appropriate times. The data checker, in addition to being turned on or off, provides BER data to the EC\&M, which is decoded to yield the total number of bits received and the total number of errors counted. 
The power meter control module allows for zeroing of the meter by removing the RF signals and looping through the zeroing command until a zero reading is obtained. The step attenuators are controlled by reading the attenuator status, calculating the next setting, and obtaining the proper command from a look-up table.

An example of a BER versus $E_{b} / N_{0}$ test sequence using an SMSK modulator and demodulator operating at a rate of $220 \mathrm{Mbps}$ which has been successfully implemented can be described. Once instruments have been zeroed and set in an initial mode, the test begins by setting the initial $E_{b} / N_{0}$ to about $5 \mathrm{~dB}$. From the theoretical curve, this will yield a BER of about $10^{-2}$. This and the next several data points can be obtained accurately with measurement times of 5 second. The sequence for measuring these points consists of determining the correct attenuator settings and measuring the $E_{b} / N_{0}$ as described above in the $E_{b} / N_{0}$ measurement section. The data generator and checker are turned on and a 5 sec wait statement is executed. The BER information is obtained from the data checker, and the program moves on to the next measurement point. Biterrors occasionaliy occur in the control word sent by the data generator. As a result, the data checker does not restart the pseudo-random data sequence properly and a 50 percent BER is erroneously measured. This problem is avoided by taking 5 samples at each measurement point and averaging the results. If a control word error has occurred in one or more of these samples, the average calculated will be close to 50 percent. If an unexpectedly bad average is noted, the bad sample(s) are found and discarded and the average recalculated, avoiding a time-consuming repeat of the measurement.

After the first 5 points have been measured, the algorithm described in Fig. 5 is implemented. For this measurement, a performance goal of $5 \times 10^{-7}$ has been set. From the theoretical curve, it was determined that with $E_{b} / N_{0}$ increments of $1 \mathrm{~dB}$, no measurement need be taken beyond a BER of 10-8 to insure at least on data point less than $5 \times 10^{-7}$. Once $10^{-8}$ has been reached, the test is stopped and the result is a measured curve such as the one shown in Fig. 1 .

The execution of a BER versus $E_{b} / N_{0}$ measurement results in a large data file which contains the status and measurement results for each step of the test sequence. Since up to 500 sequence steps may be executed during a test, two data processing programs are used to distill the data. The first program selects all of the necessary valid data values from the test data file. This includes the signal and noise power measurements and all of the BER samples at each measurement point. The $E_{b} / N_{0}$ and the $B E R$ averages are calculated and a table of $B E R$ versus $E_{b} / N_{0}$ values is printed. A second program is implemented on a desktop computer so that it can be used while BER tests are being run. This program stores the data tables on a 5-1/4 in. disk, performs anaiytical calculations such as curve fitting and BER degradation of a measured curve from the theoretical curve. The BER curves and plots of the BER degradation as a function of another measurement parameter can be plotted through this second program.

An example of this type of data reduction and plotting is shown in Fig. 8 . A series $B E R$ versus $E_{b} / N_{0}$ measurements was performed for a satellite communications system in which the input drive level of the satellite high power amplifier was successively backed off in $2 \mathrm{~dB}$ increments. The resulting BER 
curves are shown in Fig. 8(a). A clearer picture of the effect of input drive level backoff on BER can be obtained by calculating the additional $E_{b} / N_{0}$ required at the performance goal $\left(5 \times 10^{-7}\right)$ and plotting this result against the amplifier input drive level, as in Fig. 8(b).

\section{Conclusion}

A system for the efficient measurement of bit-error rates as a function of the energy-per-bit to noise power ratio has been described. The system gives accurate measurement results in a minimum amount of time. Noise insertion and $E_{b} / N_{0}$ measurement considerations and time requirements for BER measurement accuracy have been discussed. Computer control and data processing methods required for automating bit-error rate measurements have been described.

\section{References}

1. M.J. Windmiller, "Unique Bit-Error-Rate Measurement System for Satellite Communication Systems," NASA-TP-2699, 1987.

2. K.A. Shalkhauser, and G. Fujikawa, "Bit-Error-Rate Testing of High-Power 30-GHz Traveling Wave Tubes for Ground-Terminal Applications," NASA-TP-2635, 1986.

3. R.E. Ziemer, and W.H. Tranter, Principles of Communications, 2nd ed., New York: Houghton-Miffiin, 1985.

4. I. Miller, and J.E. Freund, Probability and Statistics for Engineers, 2nd ed., New York: Prentice-Hal1, 1977.

5. R.J. Kerczewsk1, and K.A. Shalkhauser, "Automated Testing of Developmental Satellite Communications Systems and Subsystems," in 25th ARFTG Conference Digest, Automatic RF Techniques Group. New York: IEEE, 1985 pp. 185-203. 


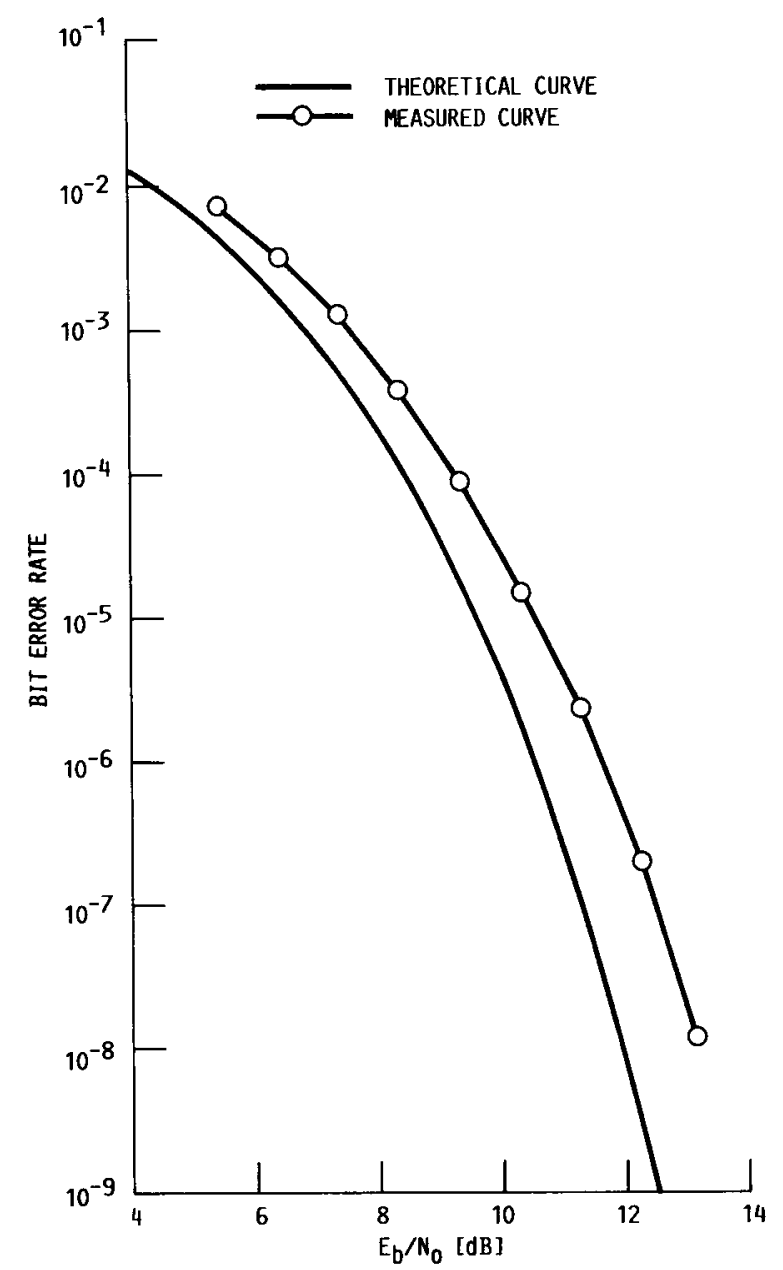

FIGURE 1. - TYPICAL MEASURED BER VERSUS $E_{b} / N_{0}$ CURVE.

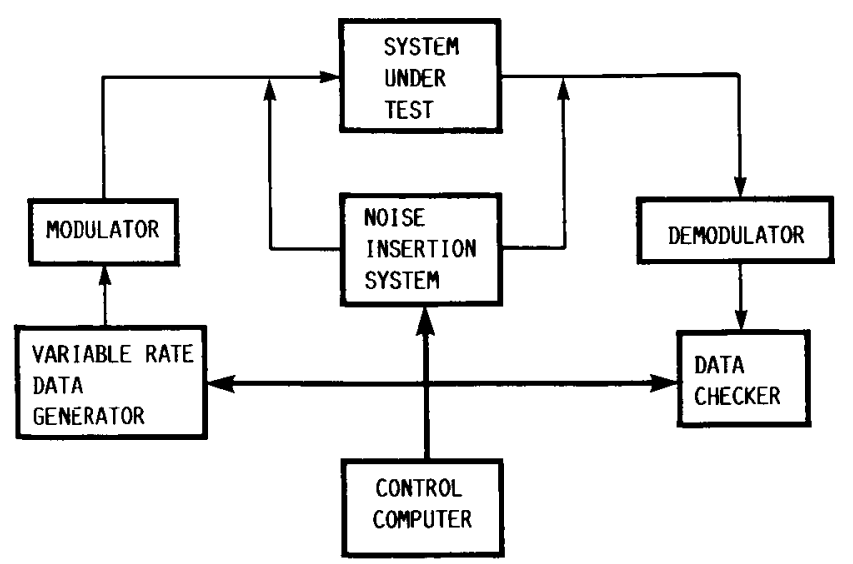

FIGURE 2. - SYSTEM FOR THE MEASUREMENT OF BIT-ERROR RATE VERSUS Eb/NO. 


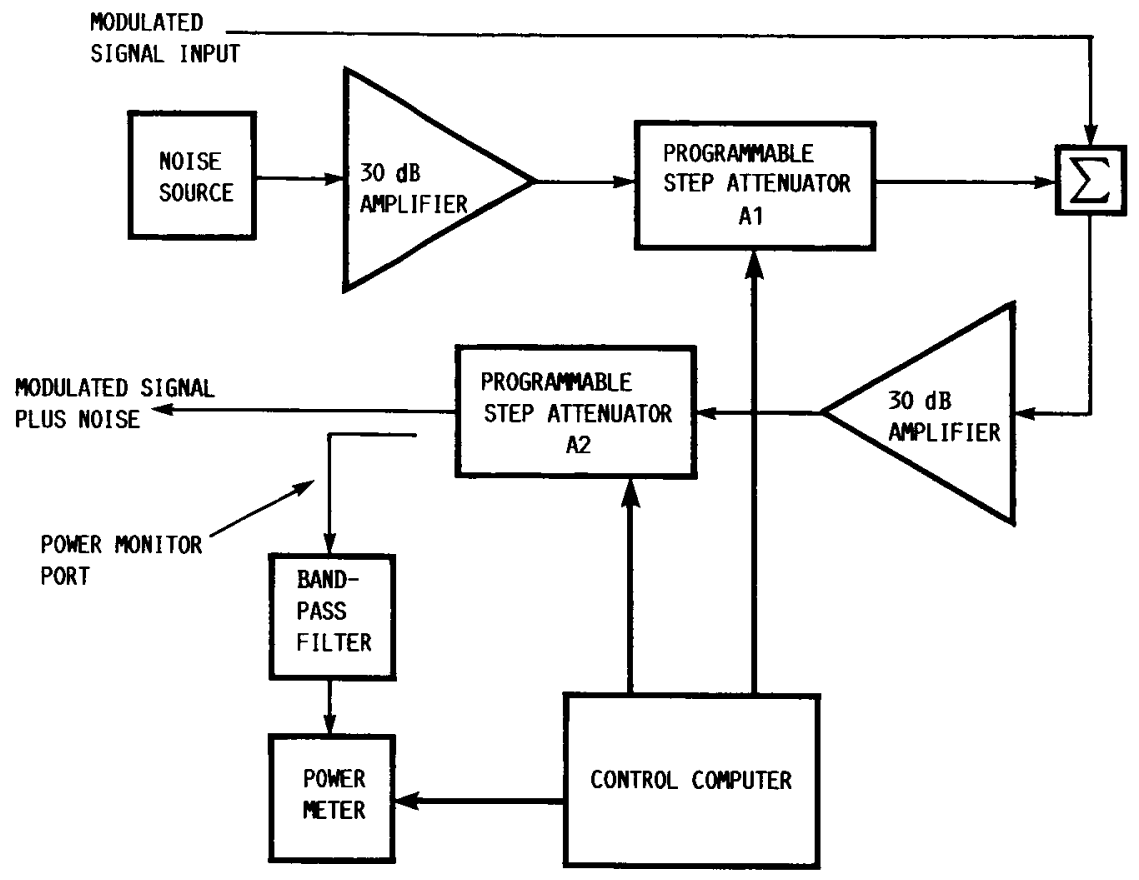

FIGURE 3. - NOISE INSERTION SYSTEM.



FIGURE 4.- NUMBER OF BITS REQUIRED VERSUS

BIT-ERROR RATE FOR THREE ERROR BOUNDS (E)

AND THREE PROBABILIJIES (P). 


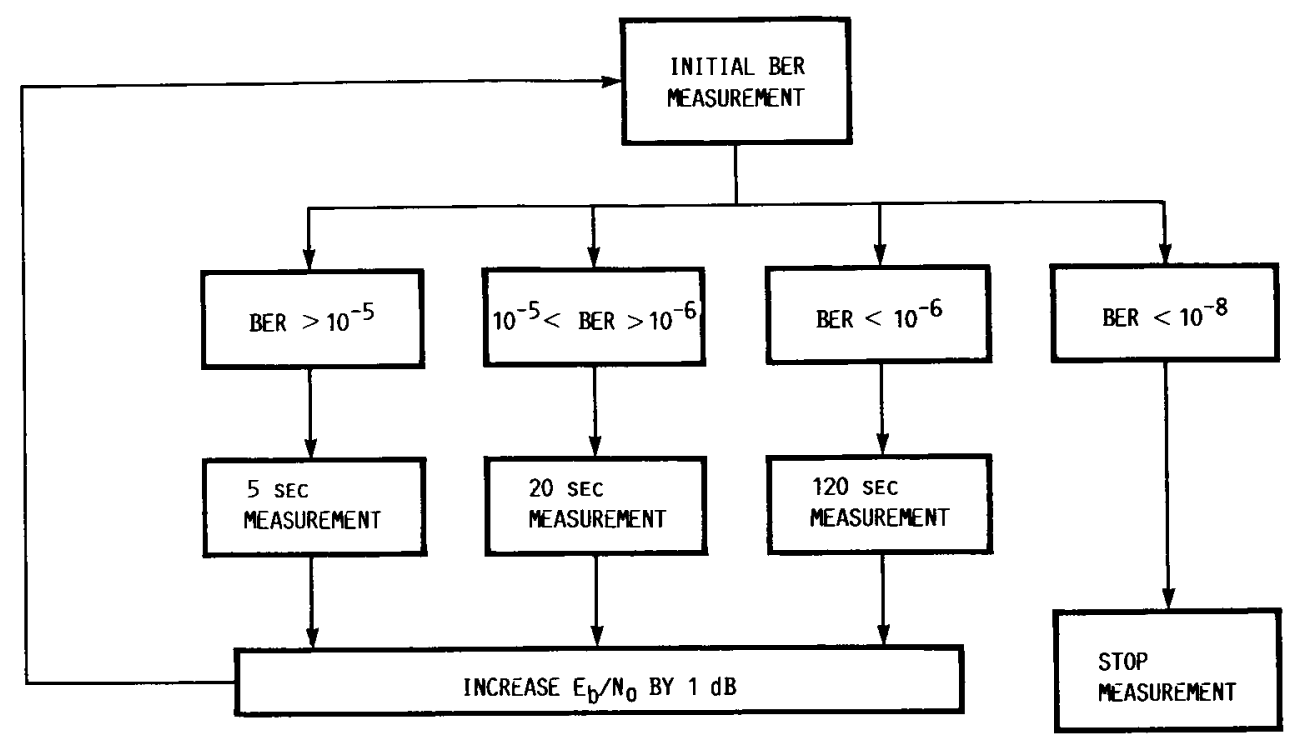

FIgURE 5. - BER MEASUREMENT TIME ALgORITHM FOR A DTA RATE OF 220 MBPS.

PHASE 1



FIgURE 6.- PHASES OF THE E C AND M CONTROL SOFTWARE.
PHASE 3
PHASE 2 




FiguRE 7. - E C AND M CONTROL PROGRAM AND MODULE LIBRARY INTERACTION. 


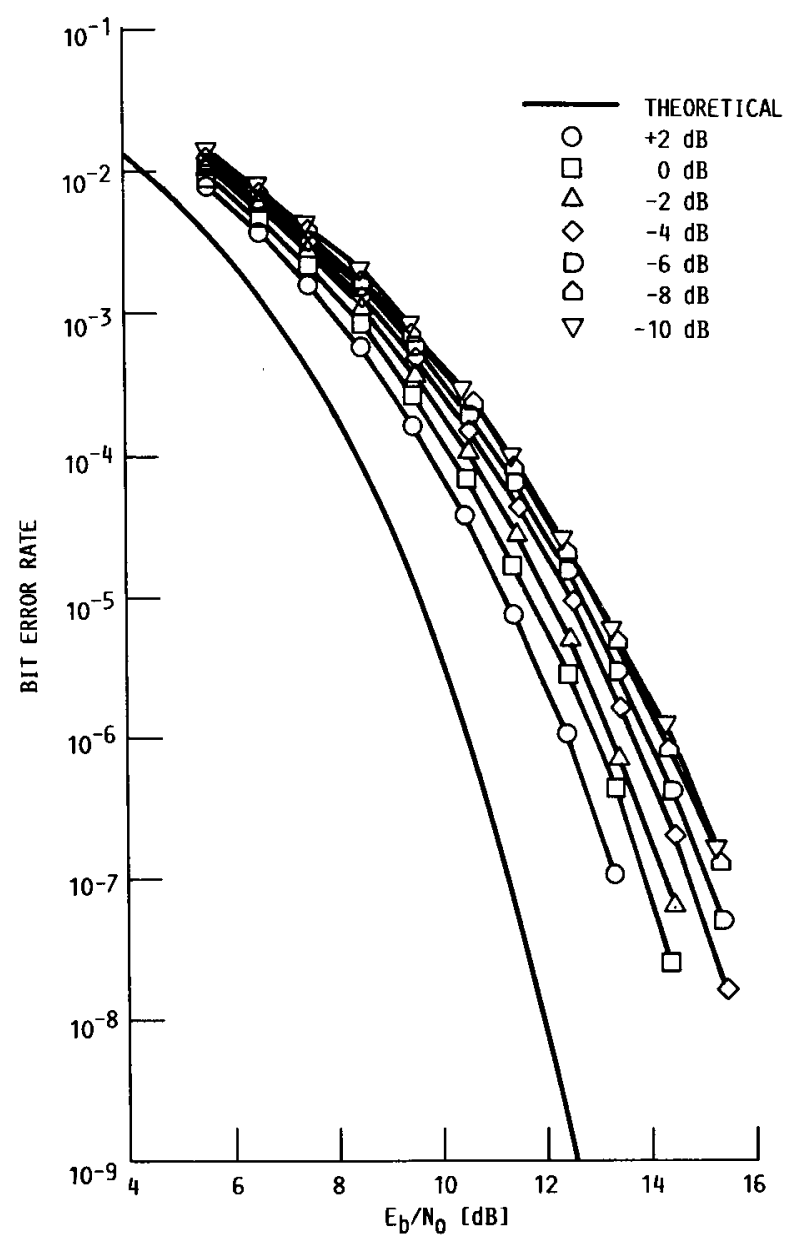

(A) BER PLOTTEd AGAINST $E_{b} / N_{0}$.

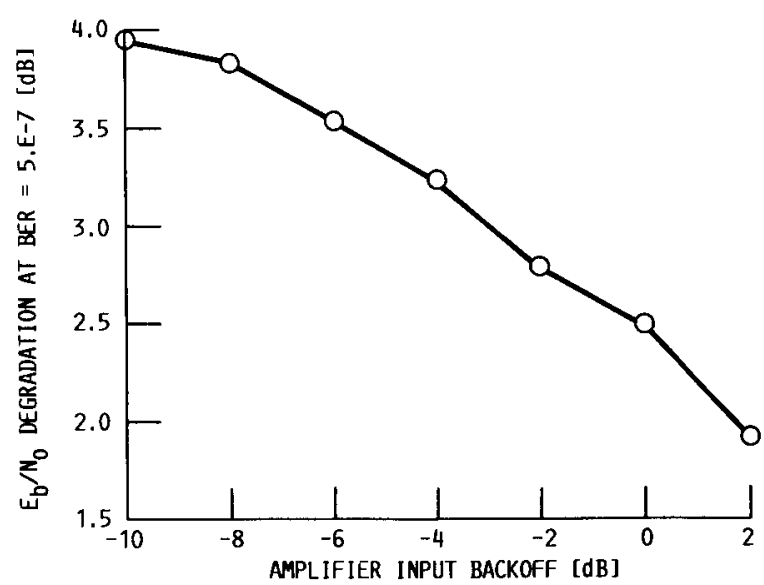

(B) DEGRADATION OF ED $/ \mathbb{N}_{0}$ FROM THE THEORETICAL CURVE PLOTTED AGAINST AMPLIFIER INPUT BACKOFF AT BER = $5 \times 10^{-7}$.

FIGURE 8. - EXAMPLE OF BER MEASUREMENTS (IN TWO PLOTTING FORMATS) FOR A SATELLITE COMMUNICATIONS LINK WHERE THE SATELLITE HIGH POWER AMPLIFIER INPUT HAS BEEN SUCCESSIVELY BACKED OFF IN $2 \mathrm{~dB}$ INCREMENTS. 


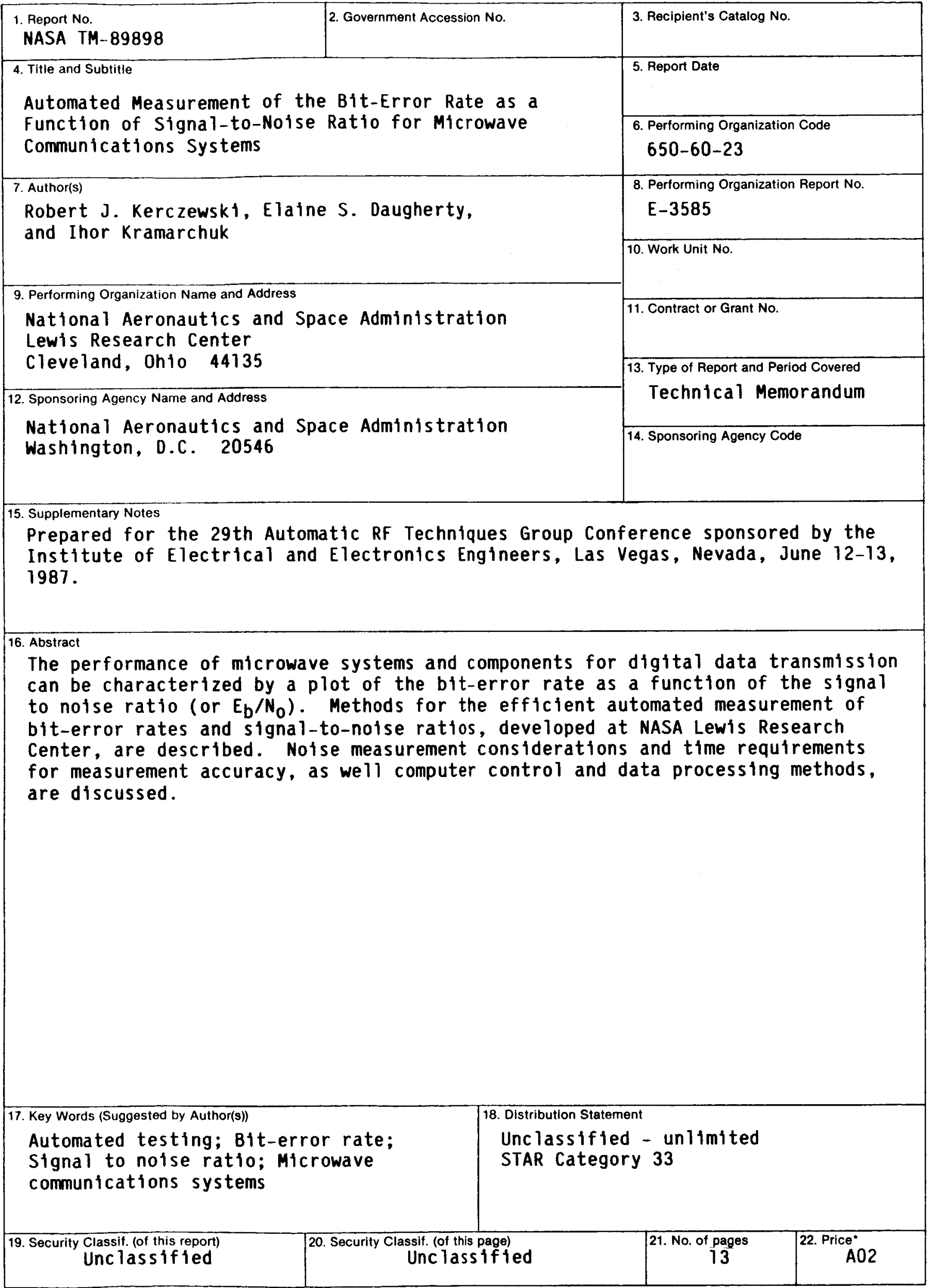

^For sale by the National Technical Information Service, Springfield, Virginia 22161 